open access WWW.ijecs.in

International Journal Of Engineering And Computer Science ISSN:2319-7242

Volume 7 Issue 12 December 2018, Page No. 24419-24421

Index Copernicus Value (2015): 58.10, 76.25 (2016) DOI: 10.18535/ijecs/v7i12.01

\title{
Real world learning ideas for effectively tutoring the basic concepts of data structure.
}

\author{
Arun Padmanabhan \\ Assistant Professor, Department of Computer Applications \\ Saintgits College of Applied Sciences \\ Kottayam, Kerala, India
}

\begin{abstract}
This paper introduces a real world learning ideas to teach the concept of data structure among the students effectively. Data structure has been the area of research for a long time in the area of technology. The need to understand the concept of data structure is thereby important not only for the students but also for the researchers.
\end{abstract}

Keywords: Data Structure, teaching style, modern technique, real world learning.

\section{Introduction}

Data structure and its concepts have to be dealt and discussed among the students effectively because of its wide range of applications. Traditional methods like classroom lecturing may have less impact as it lacks experiments and innovations. The concept of array, stack, queue and linked list can easily be taught among the students using some real world learning ideas. This teaching methodology brings more students attention and also let them understand the topics effectively. Usage of such modern teaching ideas not only empowers the student knowledge but it also improves the innovative thinking abilities of them as well. Learning through experiencing it needs to be the strategy which has to be implemented in order to teach any concepts which requires extreme thinking.

This paper introduces some interesting teaching ideas to demonstrate the ideas of data structure among students effectively.

\section{Data Structures}

\section{A. Stack}

As we know stack is a linear, non primitive and homogeneous data structure which follows LIFO terminology that is the element inserted last may be taken out first. Using any traditional teaching methods, students can only learn this concept but a more in-depth knowledge may be acquired only by attempting a new teaching experiment like below.

The back button on a web browser is an excellent example of stack implementation that is easily understood even by non experts and can easily be demonstrated in a computer lab. When a user visits a new web page, the current page gets pushed onto the stack. When the user clicks the back button, the last page pushed onto the stack is popped off the stack and loaded in the browser window. When the entire pages are deleted from the stack, the back button greys out, indicating that the stack is empty.

Note that the forward button also uses a stack, but it works on a slightly different basis. Clicking the back button pushes the current page on the stack and clicking the find button pops the top page off of the stack. Visiting a new page without clicking either button automatically inputs the forward button stack.

\section{B. Queve}

Queue is a linear, non primitive and homogenous data structure which follows FIFO terminology that is the element first inserted gets deleted first. In order to make the students learn the concept of 
queue data structure better, a novel idea like below may be useful.

During laboratory session, instruct all the students to send print requests roll number wise to a common printer connected by network. Print requests send by students will be queued in the same print queue. Later students get their job printed only in the order in which they had sent. This experiment gives the student a clear knowledge about what queue and FIFO are. Also we can show them what priority queue is by managing the job priority settings in the print options.

\section{Arrays Versus Linked List}

The arrangements as well as retrieval of data elements in computer memory when implemented using arrays or linked list are performed entirely different. For any beginner, they may found it difficult to grasp this concept. Innovative teaching ideas like below may be useful for tutors to teach their students the concept of array and linked list easily.

To teach array better, instruct students to sit roll number wise in computer lab. For example, student having roll number 5 have to take seat number 5 and student having roll number 11 thereby need to take seat number 11 accordingly. In this arrangement, no need of any address is required to locate any student. Students seated thus may easily be retrieved sequentially one after another by calling the numbers.

Unlike arrays, the linked list works on addresses. A simple experiment like below may help students to learn its concept better and effectively. Inform students to take any seat in computer lab as they wish and also to take a piece of paper (chit) in their pocket. Chit may be used to record the seat number of students in computer lab. Every student should be advised to record the seat number of his or her immediate next student as per roll number. For example, student having roll number 6 should record the seat number of student having roll number 7 . Thus if every student record his or her next counterpart in the chit, the concept of linked list is implemented. Later this experiment allow student having roll number 1 say where the next student sits using the value he or she records in his chit.
By implementing two chits in single pocket, students can easily learn the idea of doubly linked list as well. One chit may be used to record the address of his or her predecessor and other may be used to note down the address of successor as per roll number. The idea of unidirectional and bi directional traversal can also be experienced by locating the students seats using values recorded in chits.

\section{Searching And Sorting}

\section{A. Binary Search}

Binary search is an effective searching mechanism to find an element in a sorted list. Using a modern teaching methodology, teachers can bring the concept of binary search effectively among the students.

By introducing a simple among students, binary search can easily be taught. To begin the play, instruct anyone to think of a number between 1 and 80 and also to write it down without showing it to others. The job is to guess the number in the smallest number of step by making guesses by accepting hints giving in the process.

In the beginning there are 80 possible answers. Start the game by guessing number 40 , which is exactly the middle number and wait for the hint. If the hint comes is "go higher", then the range of possible correct answer is in the range of 41 to 80 . Pick the middle of that range, which is 60 . Now assume if the hint given later is "go lower", then the range of possible correct answer is in the range of 41 to 59.Amont this range, the middle number is 50.If the hint comes next is "go higher", the player goes for a number higher than 50 but lower than 60 . The game now narrowed down to a range of only nine possible numbers. Again take the number which lies in the middle location of the above range that is 55. This strategy may be follows until the player gets the right number exactly. During each guess the player should be able to narrow down the possible range of guesses by another $50 \%$. Ultimately the game is narrowed down to one answer. That in essence is how binary searches work. The word "binary" in this context by the way refers to the fact that each time, assuming we don't get right answer, we can go in two directions; to the 
left(lower) or to the right(higher). Binary means two.

\section{B. Bubble Sort}

Sorting does by swapping adjacent unordered elements until the entire list is sorted either in ascending or descending order. An innovative idea like below may be used to teach this sorting better among students.

Form a random line when teachers are asked during an assembly. The bubble sort algorithm comes in handy here to arrange the students in increasing or decreasing order of height.

In this case every person's height is an element of the list. With every pass that the teacher goes over the students, they slowly start standing in a more orderly fashion till all of them stand according to height.

\section{CONCLUSION}

Modern teaching ideas discussed above may be beneficial to share the concept of data structure among students effectively and easily. Traditional teaching methods like classroom lecturing, memorization and recitation techniques may not be much beneficial to teach complex concepts of data structures. Modern teaching technique discussed here is "Real - World learning", which infuses real life experiences into your instructions which will make teaching moments fresh, and enrich classroom teaching.

Relating and demonstrating through real-life situations will make the material easy to understand and easy to learn. It will spark their interest and get the children excited and involved.

\section{References}

[1] Ashok N. Kamthane. 2007 Introduction to Data Structures in C. Pearson Education. 